\title{
Surgical Experience in Cases of L5 and S1 Symptoms Caused by Upper Lumbar Spinal Stenosis of L2 - L3 and L3 - L4
}

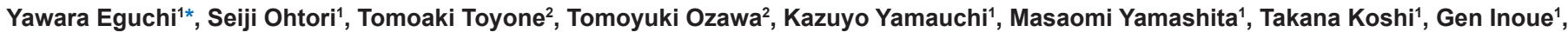
Munetaka Suzuki ${ }^{1}$, Sumihisa Orita ${ }^{1}$, Hiroto Kamoda ${ }^{1}$, Gen Arai ${ }^{1}$, Tetsuhiro Ishikawa ${ }^{1}$, Masayuki Miyagi ${ }^{1}$, Yasuchika Aoki ${ }^{3}$ and Kazuhisa Takahashi $^{1}$

${ }^{1}$ Department of Orthopaedic Surgery, Graduate School of Medicine, Chiba University, 1-8-1 Inohana, Chuo-ku, Chiba, Japan

${ }^{2}$ Department of Orthopaedic Surgery, Teikyo University Chiba Medical Center, Chiba, Japan

${ }^{3}$ Department of Orthopedic Surgery, Toho University Sakura Medical Center, Chiba, Japan

\section{Abstract}

Object: Previous reports have indicated that the level of lumbar spinal canal stenosis (LSCS) often differs from that diagnosed from neurological symptoms, and L5 nerve roots are often affected by stenosis at the L2-L3 or L3-L4 level; however, few cases have been described.

Decompression surgery for upper lumbar spinal canal stenosis (LSCS) of L2-L3 and L3-L4 causing L5 and S1 symptoms was investigated.

Methods: Eight patients with a diagnosis based on L5 or S1 symptoms, but whose MRI or CT-myelography showed only one level of stenosis at L2-L3 or L3-L4 were studied. The level of stenosis was determined by the most narrowing lesion, such as total or subtotal block on CT-myelography and MRI. Selective nerve root block was performed to determine which nerve root was the origin of the pain in these patients. One-level decompression surgery at L2-L3 or L3-L4 was performed in 8 patients.

Results: There were 2 cases of stenosis at L2-L3 and 6 cases at L3-L4. The level involved suggested by neurological symptoms was L5 in 6 cases and S1 in 2 cases. L5 symptoms were most often affected by L3-L4. Symptoms in all patients disappeared after one-level decompression surgery.

Conclusions: Degenerative stenosis of upper levels such as L2-3 and L3-4 involved damage in lower nerve roots such as L5 or S1, and L5 symptoms were most often affected by L3-L4. Decompression surgery for upperlevel stenosis improved symptoms in all patients. Physicians should be aware that upper-level stenosis can cause radiculopathy at a lower level.

Keywords: Lumbar spinal stenosis; Surgical treatment; Cauda equine; Root compression

\section{Introduction}

Verbiest described that developmental narrowing of the lumbar vertebral canal causes radicular syndrome [1]. The neurological findings tend to be aggravated with severity of the lumbar spinal canal stenosis (LSCS). Radicular symptoms are usually related to the level of the responsible lesion; the L5 root is compressed in the L4-L5 space or foraminal or extraforaminal level of the L5-S1 space. In contrast, compression in the L3-L4 space affects the L4 root. However, in some patients the level of stenosis on the image does not correlate with the neurological findings. Previous reports have indicated that the level of LSCS often differs from that diagnosed from neurological symptoms, and L5 nerve roots were often affected by stenosis at the L1-L2 or L2L3 level [2-5]. The cauda equina are bundles of nerve roots within the thecal sac and at the L3-L4 intervertebral level, the L4 root is situated anterolaterally and the lower roots such as L5 and S1 roots tend to take up a more medial central position (Figure 1A) [6,7]. According to the specific anatomical structure of the lumbar thecal sac, a severe compression of the thecal sac at the L3-L4 level may damage the caudal roots such as the L5 and S1 roots (Figure 1B). The purpose of this study was to perform one-level decompression for patients whose nerve root symptoms indicated L5 or S1 involvement, but whose magnetic resonance imaging (MRI) or computed tomographic (CT) myelograms indicated only L2-L3 or L3-L4 stenosis.

\section{Materials and Methods}

\section{Inclusion and exclusion criteria}

This study was approved by our Institutional Ethics Committee and informed consent was obtained from the participants. From June 2003 to April 2009, a total of 364 patients with LSCS who underwent lumbar spine surgical treatment were studied. Of the 364 patients, 8 patients
A

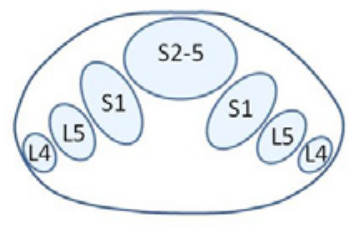

L3-4
B

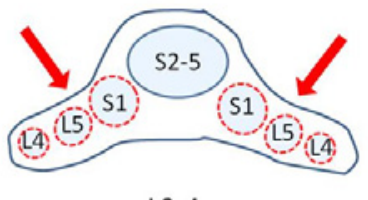

L3-4
Figure 1: An anatomical diagram of the cauda equina within the thecal sac at the L3-L4 intervertebral level. A. The L4 root situated anterolaterally and the lower roots such as L5 and S1 roots tend to take up a more medial central position. B. A severe compression of the thecal sac at the L3-L4 level may damage caudal roots such as the L5 and S1 roots.

*Corresponding author: Yawara Eguchi, MD, PhD, Department of Orthopaedic Surgery, Graduate School of Medicine, Chiba University, 1-8-1 Inohana, Chuoku, Chiba, 260-8670, Japan, Tel: +81-43-226-2117; Fax: +81-43-2116; E-mail: yawara_eguchi@yahoo.co.jp

Received November 10, 2011; Accepted December 16, 2011; Published December 19, 2011

Citation: Eguchi Y, Ohtori S, Toyone T, Ozawa T, Yamauchi K, et al. (2012) Surgical Experience in Cases of $L 5$ and S1 Symptoms Caused by Upper Lumbar Spinal Stenosis of L2-L3 and L3-L4. J Spine 1:105. doi:10.4172/2165-7939.1000105

Copyright: ( 2012 Eguchi Y, et al. This is an open-access article distributed under the terms of the Creative Commons Attribution License, which permits unrestricted use, distribution, and reproduction in any medium, provided the original author and source are credited. 
(6 men, 2 women, average age 59.6 years) with a diagnosis based on symptoms of L5 or S1, but whose MRI and CT-myelography showed only one level of stenosis at L2-L3 or L3-L4 were suitable for inclusion in this study. The patient exclusion criteria were follows: (1) those that had stenosis at other levels, (2) those that had foraminal stenosis, (3) those that had lumbar spine surgery before this study, and (4) those that had transitional vertebra. The level of neurological disturbance was determined by neurological examinations and included deficit of the musculature, hypoesthesia, leg pain, osteotendious reflexes. The sensory disturbance was evaluated using a dermatome described by Keegan [8]. Quadriceps muscle weakness was found because of both L3 and L4 radiculopathies, extensor hallucis longus weakness because of L5 radiculopathy, and flexor hallucis longus and gastrocnemius weakness because of S1 radiculopathy [9]. The level of stenosis on the image was determined by the most narrowing lesion such as total block or subtotal block on the CT-myelography and MRI. Selective nerve-root block with lidocaine $(1 \%, 1 \mathrm{ml})$ was performed to ultimately determine which nerve root was the origin of the pain in these patients. L3, L4, L5, and S1 root blocks were performed in cases of L2-L3 stenosis, while L4, L5, and S1 root blocks were used in cases of L3-L4 stenosis. The patients were blinded with regard to each of the blocks, which were performed on different days. The selective nerve-root block was defined as effective when leg pain VAS score was reduced more than $60 \%$ by the block. One-level decompression surgery at L2-L3 or L3-L4 was performed in 8 patients. The diagnoses and surgical treatment of patients were performed by five trained spine surgeons.

\section{CT-myelography protocol}

All subjects underwent CT-myelography (Aquilion 64, Toshiba Medical, Tochigi, Japan) with a tube voltage of $120 \mathrm{kV}$, tube current of $450 \mathrm{~mA}$ (CT-AEC), 0.5 s/rotation and helical pitch of 0.83 . Ten milliliters of contrast medium (iohexol: $240 \mathrm{mg} / \mathrm{ml}$, Tokyo, Japan) was injected into the subarachnoid space before scanning. The patients were rolled to ensure that the contrast medium was completely distributed.

\section{MRI protocol}

A 1.5-T MRI scanner (Philips Medical systems) was used in this study. Sagittal T1-weighted (TR/TE, 400/14), axial and sagittal T2weighted fast spin-echo (TR/TE, 4000/102) sequences were obtained using a $256 \times 256$ matrix, $260 \mathrm{~mm}$ field of view (FOV), and 3/1 mm slice thickness/gap.

\section{Evaluation of physical examinations and patient pain scores}

The level of stenosis, presence of spondylolisthesis, level of neurological disturbance detected by selective nerve root block, distance of intermittent claudication (IMC), straight-leg-raising (SLR), femoral-nerve-stretch test (FNST), and the deficit of the musculature were investigated. Clinical results were assessed using the visual analogue scale (VAS) score of low-back pain (LBP), leg pain from 100 (extreme amount of pain) to zero (no pain), and Japanese Orthopedic Association (JOA) scoring system (Table 1). The normal score is 29 points, based on 3 subjective symptoms (9 points), 3 clinical signs including straight-leg raising (6 points), and 7 activities of daily living (14 points). The clinical evaluations were conducted before surgery and at 12 months after surgery.

\section{Results}

Table 2 shows a summary of the levels of stenosis, physical examinations, and clinical results. There were 2 cases of stenosis at
L2-L3 and 6 cases at L3-L4. There were 5 cases of subtotal block and 3 cases of total block on CT-myelography. The level on the basis of neurological symptoms was L5 in 6 cases and S1 in 2 cases. L5 or S1 root blocks of the responsible lesion reduced leg pain effectively, but

\begin{tabular}{|c|c|c|c|}
\hline \multicolumn{3}{|l|}{ Subjective symptoms Score } & \\
\hline & \multicolumn{2}{|c|}{$\begin{array}{l}\text { (Maximum } \\
9 \text { points) }\end{array}$} & \\
\hline \multicolumn{4}{|l|}{ Low-back pain } \\
\hline None & 3 & & \\
\hline Occasional mild pain & 2 & & \\
\hline Frequent mild or occasional severe pain & 1 & & \\
\hline Frequent or continuous severe pain & 0 & & \\
\hline \multicolumn{4}{|l|}{ Leg pain or tingling } \\
\hline None & 3 & & \\
\hline Occasional slight symptoms & 2 & & \\
\hline Frequent slight or occasional symptoms & 1 & & \\
\hline Frequent or continuous severe symptoms & 0 & & \\
\hline \multicolumn{4}{|l|}{ Gait } \\
\hline Normal & 3 & & \\
\hline $\begin{array}{l}\text { Able to walk farther than } 500 \mathrm{~m} \text { although resulting in pain, } \\
\text { tingling, or muscle weakness }\end{array}$ & 2 & & \\
\hline $\begin{array}{l}\text { Unable to walk farther than } 500 \mathrm{~m} \text { because of leg pain, } \\
\text { tingling, or muscle weakness }\end{array}$ & 1 & & \\
\hline $\begin{array}{l}\text { Unable to walk farther than } 100 \mathrm{~m} \text { because of leg pain, } \\
\text { tingling, or muscle weakness }\end{array}$ & 0 & & \\
\hline Clinical signs & \multicolumn{2}{|c|}{$\begin{array}{l}\text { (Maximum } \\
6 \text { points) }\end{array}$} & \\
\hline \multicolumn{4}{|l|}{ Straight-leg-raising (including tight hamstrings) } \\
\hline Normal & 2 & & \\
\hline $30^{\circ}$ to $70^{\circ}$ & 1 & & \\
\hline Less than $30^{\circ}$ & 0 & & \\
\hline \multicolumn{4}{|l|}{ Sensory disturbance } \\
\hline None & 2 & & \\
\hline Slight disturbance (not subjective) & 1 & & \\
\hline Marked disturbance & 0 & & \\
\hline \multicolumn{4}{|l|}{ Motor disturbance (MRC grade) } \\
\hline None (5) & 2 & & \\
\hline Slight disturbance (4) & 1 & & \\
\hline Marked disturbance (3 to 0 ) & 0 & & \\
\hline \multirow[t]{3}{*}{ Activities of daily living } & $(\operatorname{Max}$ & imum & \\
\hline & \multicolumn{3}{|c|}{ Restrictions } \\
\hline & \multicolumn{3}{|c|}{$\begin{array}{l}\text { Severe } \\
\text { Moderate None }\end{array}$} \\
\hline Turning over while lying & 0 & 1 & 2 \\
\hline Standing & 0 & 1 & 2 \\
\hline Washing & 0 & 1 & 2 \\
\hline Leaning forward & 0 & 1 & 2 \\
\hline Sitting (about 1 hour) & 0 & 1 & 2 \\
\hline Lifting or holding heavy objects & 0 & 1 & 2 \\
\hline Walking & 0 & 1 & 2 \\
\hline Urinary bladder function & $(\operatorname{Max}$ & imum & \\
\hline Normal & 0 & & \\
\hline Mild dysuria & -3 & & \\
\hline Severe dysuria & -6 & & \\
\hline
\end{tabular}

Table 1: Japanese Orthopaedic Association (JOA) Scoring System for Assessment of Low-Back Pain. 
Citation: Eguchi Y, Ohtori S, Toyone T, Ozawa T, Yamauchi K, et al. (2012) Surgical Experience in Cases of L5 and S1 Symptoms Caused by Upper Lumbar Spinal Stenosis of L2-L3 and L3-L4. J Spine 1:105. doi:10.4172/2165-7939.1000105

Page 3 of 4

\begin{tabular}{|c|c|c|c|c|c|c|c|c|c|c|c|c|c|c|c|c|c|}
\hline \multirow{3}{*}{$\begin{array}{c}\text { No. } \\
1\end{array}$} & \multirow{3}{*}{$\begin{array}{c}\text { Age(y) } \\
48\end{array}$} & \multirow{3}{*}{$\begin{array}{c}\text { Gender } \\
\mathrm{F}\end{array}$} & \multirow{2}{*}{\multicolumn{2}{|c|}{$\begin{array}{l}\text { Level of } \\
\text { stenosis }\end{array}$}} & \multirow{3}{*}{$\begin{array}{c}\text { Spondyl- } \\
\text { olisthesis } \\
+\end{array}$} & \multirow{3}{*}{$\begin{array}{c}\begin{array}{c}\text { Neurological } \\
\text { disturbance }\end{array} \\
\text { L5 Bil } \\
\end{array}$} & \multirow{3}{*}{\begin{tabular}{|c|} 
IMC (m) \\
100
\end{tabular}} & \multirow{3}{*}{\begin{tabular}{|c|} 
SLR \\
+
\end{tabular}} & \multirow{3}{*}{$\begin{array}{c}\text { FNST } \\
-\end{array}$} & \multirow{3}{*}{$\begin{array}{c}\begin{array}{c}\text { Motor } \\
\text { deficit }\end{array} \\
E H L\end{array}$} & \multirow{3}{*}{$\begin{array}{c}\begin{array}{c}\text { Surgical } \\
\text { method }\end{array} \\
\text { ALIF }\end{array}$} & \multirow{3}{*}{$\begin{array}{c}\text { JOA } \\
\text { (Pre) }\end{array}$} & \multirow{3}{*}{$\begin{array}{c}\begin{array}{c}\text { JOA } \\
\text { (Post) }\end{array} \\
11\end{array}$} & \multicolumn{2}{|c|}{ VAS (Pre) } & \multicolumn{2}{|c|}{ VAS (Post) } \\
\hline & & & & & & & & & & & & & & \multirow{2}{*}{$\begin{array}{r}\text { LBP } \\
60\end{array}$} & \multirow{2}{*}{\begin{tabular}{|c|}
$\begin{array}{c}\text { Leg } \\
\text { pain }\end{array}$ \\
90
\end{tabular}} & \multirow{2}{*}{$\begin{array}{c}\text { LBP } \\
20\end{array}$} & \multirow{2}{*}{$\begin{array}{c}\begin{array}{c}\text { Leg } \\
\text { pain }\end{array} \\
50\end{array}$} \\
\hline & & & $\mathrm{L} 3 / 4$ & subtotal & & & & & & & & & & & & & \\
\hline 2 & 46 & M & L2/3 & subtotal & - & L5 Bil & - & + & - & - & ALIF & 15 & 26 & 60 & 65 & 20 & 20 \\
\hline 3 & 68 & $M$ & $L 3 / 4$ & subtotal & + & S1 Bil & 700 & - & - & EHL & PLF & 19 & 27 & 50 & 75 & 30 & 10 \\
\hline 4 & 55 & $\mathrm{~F}$ & $\mathrm{~L} 3 / 4$ & total & + & L5 Bil & 10 & + & + & TA, EHL & ALIF & 9 & 26 & 60 & 95 & 20 & 10 \\
\hline 5 & 68 & $\mathrm{M}$ & $L 3 / 4$ & subtotal & + & L5 Lt & 500 & - & - & - & PLF & 17 & 25 & 20 & 85 & 10 & 0 \\
\hline 6 & 73 & M & $\mathrm{L} 3 / 4$ & total & + & L5 Lt & 50 & - & - & EHL & PLF & 9 & 24 & 50 & 92 & 30 & 10 \\
\hline 7 & 57 & M & $\mathrm{L} 2 / 3$ & subtotal & - & $\mathrm{L} 5 \mathrm{Lt}$ & 500 & - & - & EHL & laminectomy & 10 & 25 & 70 & 90 & 10 & 0 \\
\hline 8 & 62 & M & $\mathrm{L} 3 / 4$ & total & + & S1 Bil & 100 & - & - & - & TILF & 9 & 25 & 50 & 85 & 20 & 25 \\
\hline Mean & 59.6 & & & & $75 \%$ & & 280 & $37 \%$ & $12 \%$ & $60 \%$ & & 11.5 & 23.6 & 52.5 & 84.6 & 20 & 15.6 \\
\hline
\end{tabular}

Table 2:

L3 or L4 root blocks did not. L5 symptoms were most often affected by L3-L4. Plain radiographs showed 6 cases (75\% of 8 patients) of spondylolisthesis. Physical examinations revealed that the mean distance for intermittent claudication was $280 \mathrm{~m}$, a positive SLR test greater than $70^{\circ}$ in 3 cases (37\%), a positive FNST test in one case (12\%), and motor weakness (4 cases of extensor hallucis longus and one case of tibialis anterior and extensor hallucis longs) in 5 cases (60\%). One anterior lumbar interbody fusion (ALIF) and one laminectomy were performed for L2-L3. Three posterolateral fusions (PLFs), 2 ALIFs, and one transforaminal lumbar interbody fusion (TLIF) were performed for L3-L4. LBP VAS score was improved from 52.5 points to 20 points, leg pain VAS score from 84.6 points to 15.6 points, and JOA score from 11.5 points to 23.6 points after surgery.

\section{Case presentation}

A 68-year-old man (case 5 in Table 2) experienced a sudden onset of low-back pain and left calf pain in the fifth lumbar dermatome after heavy lifting. His pain and numbness worsened and he had claudication of $500 \mathrm{~m}$ after beginning ambulation for 2 months before being admitted to our hospital. Physical examination showed no motor weakness or hypoesthesia of his lower extremities bilaterally. An SLR test and FNST were normal. His lower extremity reflexes were normal. A rectal examination was normal. The laboratory findings were normal. The plain radiographs showed spondylolisthesis with $20 \%$ anterior slip of the third lumbar vertebra. Myelograms showed subtotal block at L3L4. CT-myelography and MRI showed severe stenosis at L3-L4, but no stenosis at L4-L5 or L5-S1 (Figure 2). Selective fifth nerve root block, performed using $1 \mathrm{ml}$ of $1 \%$ lidocaine, relieved the pain in the left calf, but a fourth nerve root block did not.

The patient continued to suffer pain in spite of treatment, so a L3L4 laminotomy and posterolateral fusion were performed (Figure 3B). A thickened ligamentum flavum that compressed the dural sac on the left dorsal side was extracted (Figure $3 \mathrm{~A}$ ). At the end of the procedure, the dural sac was unobstructed and the L4 roots were not compressed.

The postoperative evaluation of the patient was satisfactory. His JOA score was improved from 17 points to 25 points, LBP VAS score from 20 points to 10 points, and leg pain VAS score from 85 points to 0 points. One year later, he was free of symptoms.

\section{Discussion}

Radicular symptoms are usually related to the level of the responsible lesion; L5 roots are compressed in the L4-L5 space or
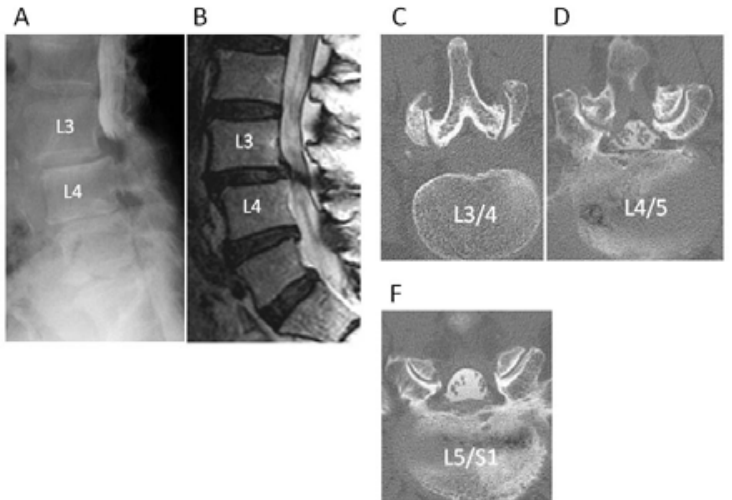

Figure 2: A 68-year-old man with low-back pain and L5 radiculopathy at his left calf. A. Sagittal myelogram showing subtotal block at L3-L4. B. Sagittal MRI showing severe stenosis at L3-L4, but no stenosis at L4-L5 or L5-S1. C-D. Axial CT-myelography showing severe stenosis at L3-L4, but no stenosis at L4-L5 or L5-S1.
A

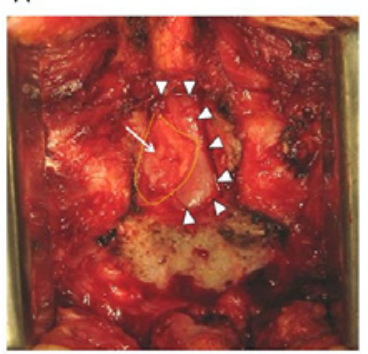

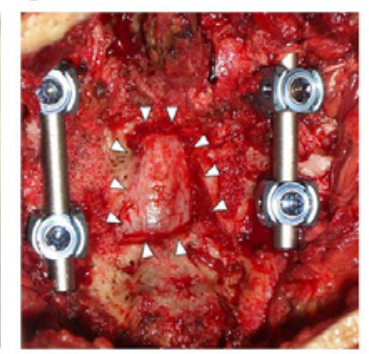

Figure 3: Only L3-L4 laminotomy and posterolateral fusion were performed. A. A thick ligamentum flavum that compressed the dural sac on the left dorsal side was extracted. The arrow and dotted line circle indicate the thick ligamentum flavum. Arrowheads show the dural sac. B. At the end of the procedure, the dural sac was unobstructed and the L4 roots were not compressed. The arrowhead shows the dural sac.

foraminal or extraforaminal level of the L5-S1 space producing calf pain or paresthesia in the root-dependent area or extensor hallucis longus weakness or a positive straight-leg-raising test. In contrast, compression of the L2-L3 and L3-L4 spaces affects the L3 and L4 roots, producing anterior thigh pain, quadriceps muscle weakness, or a positive femoral-nerve-stretch test or weakness of the patella reflex. However, reports of previous studies [2-5] indicated that L5 nerve 
roots were often affected by stenosis at the L1-L2 or L2-L3 levels. If the level of LSCS differs from that diagnosed from neurological symptoms, it is difficult to determine which spaces are producing the clinical symptoms and should be decompressed. Compression at the thoracolumbar level or at the first or second lumbar level produces complex symptoms because of the proximity of the medullary epicone and cone and cauda equina, although mostly the level of L1-L2 was more cranial than the level of L2-L3 and L3-L4 in our study.

We showed that the LSCS of a single upper level such as L2-L3 and L3-L4 involved damage to a lower nerve root such as L5 or S1. Decompression and fusion surgery for upper-level stenosis improved symptoms in all patients. In this study, no stenosis other than that at upper spaces existed; however, if there was stenosis at a lower space or foraminal stenosis, it was difficult to determine which levels produced the clinical symptoms. MRI in asymptomatic individuals may show false positive findings. An evaluation by Boden et al. [10] found that $57 \%$ of asymptomatic subjects who were over 60 years of age were found to have abnormal findings. Many imaging techniques, including plain radiography, CT, myelograms, CT myelograms, MRI, and selective nerve root block have been needed to diagnose the level responsible for the clinical symptoms.

Olmarker et al. [11] reported that impairment of impulse propagation in pig cauda equina was proportional to the pressure of applied compression, that double-level compression induced more pronounced effects on nerve propagation than single-level compression [12], and that edema and demyelination in spinal nerve roots were more pronounced after rapid than after slow onset of compression $[13,14]$. Sato et al. [15] demonstrated that the cauda equina and L5 nerve roots were more often affected than the L4 nerve roots in patients with a two-level stenosis. These results indicated that severe LSCS induced more pronounced damage of cauda equina nerve roots and multi-level stenosis induced more severe damage in lower nerve roots compared with single-level stenosis.

Several explanations could account for the stenosis of an upper level being involved in damage to a lower nerve root. Firstly, severe upper-level stenosis may affect the entire lower cauda equina as described in the introduction. Second, in Porter's hypothesis [16], a two-level central canal stenosis causes venous congestion and pooling between the two levels of compression, which leads to an inadequate oxygen supply in the nerve roots of the cauda equina. Moreover, on myeloscopic examination, the diameters of vessels of the cauda equina have shown significant changes in patients in accordance with postural alteration [17]. Govind [18] reported that radicular pain is caused by a mass effect: the combination of mechanical stimulation, inflammation, and ischemic damage resulting from intraneural edema and demyelination has the potential to generate ectopic impulses from the affected nerve. The venous congestion of cauda equina by upperlevel compression may damage the lower nerve roots including the dorsal root ganglia and cause radiculopathy at a lower level. Third, because both L3 and L4 nerve roots control the quadriceps, quadriceps muscle weakness does not tend to occur because of L3 or L4 monoradiculopathy, but the $\mathrm{L} 5$ nerve root controls single muscles such as the extensor hallucis longus, so that prominent symptoms such as muscle weakness are obvious in L5 radiculopathy [19]. Fourth, nerve root anomalies such as the furcal nerve which gives branches to L4 and L5 root may cause atypical neurological findings [20].

We acknowledge that our study is limited by the small number of subjects investigated. Further studies are needed to investigate whether our findings are valid in the broader population.

In conclusion, this study showed that LSCS of an upper level such as L2-L3 and L3-L4 involved damage to a lower nerve root such as those at L5 or S1, L5 symptoms were most often affected by L3-L4, and decompression and fusion surgery for upper level stenosis improved symptoms. Physicians should be aware that upper-level stenosis can cause radiculopathy at a lower level.

\section{References}

1. Verbiest $H$ (1954) A radicular syndrome from developmental narrowing of the lumbar vertebral canal. J Bone Joint Surg Br 36: 230-237.

2. Shirado O, Matsukawa S, Kaneda K (1996) Herniation of the disc between the first and second lumbar vertebrae with a monoradiculopathy of the fifth lumbar nerve root. J Bone Joint Surg Am 78: 1422-1426.

3. Korovessis P, Baikousis A, Stamatakis M, Katonis P (1998) Monoradiculopathy of the fifth lumbar nerve root due to lumbar disc herniation between lumbar one and lumbar two vertebrae. J Spinal Disord 11: 350-353.

4. Hidalgo-Ovejero AM, García-Mata S, Martínez-Grande M (1998) L5 roo compression caused by degenerative spinal stenosis. Spine 17: 1268-1272.

5. Hidalgo-Ovejero AM, García-Mata S, Sánchez-Villares JJ, Lasanta P, IzcoCabezon T, et al. (2003) L5 root compression resulting from an L2-L3 disc herniation. Am J Orthop 32: 392-394.

6. Cohen MS, Wall EJ, Kerber CW, Abitbol JJ, Garfin SR (1991) The anatomy of the cauda equina on CT scans and MRI. J Bone Joint Surg Br 73: 381-384.

7. Wall EJ, Cohen MS, Massie JB, Rydevik B, Garfin SR (1990) Cauda equina anatomy. I: Intrathecal nerve root organization. Spine 15: 1244-1247.

8. Keegan JJ (1944) Neurosurgical interpretation of dermatome hypalgesia with herniation of the lumbar intervertebral disc. J Bone Joint Surg Am 26: 238-248.

9. Haymaker W, Woodhall B (1953) Peripheral nerve injures: principles and diagnosis. Philadelphia: WB Saunders.

10. Boden SD, Davis DO, Dina TS, Patronas NJ, Wiesel SW (1990) Abnorma magnetic-resonance scans of the lumbar spine in asymptomatic subjects: A prospective investigation. J Bone Joint Surg Am 72: 403-408.

11. Olmarker K, Holm S, Rydevik B (1990) Importance of compression onse rate for the degree of impairment of impulse propagation in experimental compression injury of the porcine cauda equina. Spine 15: 416-419.

12. Olmarker K, Rydevik B (1992) Single-versus double-level nerve root compression. An experimental study on the porcine cauda equina with analyses of nerve impulse conduction properties. Clin Orthop Relat Res 279: 35-39.

13. Olmarker K, Rydevik B, Holm S (1989) Edema formation in spinal nerve roots induced by experimental, graded compression: an experimental study on the pig cauda equina with special reference to differences in effects between rapid and slow onset of compression. Spine 14: 569-573.

14. Cornefjord M, Sato K, Olmarker K, Rydevik B, Nordborg C (1997) A mode for chronic nerve root compression studies. Presentation of a porcine model for controlled, slow-onset compression with analyses of anatomic aspects, compression onset rate, and morphologic and neurophysiologic effects. Spine 22: $946-957$

15. Sato K, Kikuchi S (1997) Clinical analysis of two-level compression of the cauda equina and the nerve roots in lumbar spinal canal stenosis. Spine 22 1898-1903

16. Porter RW, Ward D (1992) Cauda equina dysfunction: The significance of twolevel pathology. Spine 17: 9-15

17. Ooi Y, Mita F, Satoh Y (1990) Myeloscopic study on lumbar spinal canal stenosis with special reference to intermittent claudication. Spine 15: 544-549.

18. Govind J (2004) Lumbar radicular pain. Aust Fam Physician 33: 409-412.

19. Hirabayashi H, Takahashi J, Hashidate H, Ogihara N, Tashiro A, et al. (2009) Characteristics of L3 nerve root radiculopathy. Surg Neurol 72: 36-40.

20. Kikuchi S, Hasue M, Nishiyama K, Ito T (1986) Anatomic features of the furcal nerve and its clinical significance. Spine 11: 1002-1007. 